\title{
Past and Present Secular Evolution in the Host Galaxies of NLS1s
}

\author{
G. Orban de Xivry ${ }^{* i a}$, R. Davies ${ }^{a}$, M. Schartmann ${ }^{a, b}$, S. Komossa ${ }^{a, c, d, e}$, A. Marconi ${ }^{f}$, \\ E. Hicks ${ }^{g}$, H. Engel ${ }^{a}$, L. Tacconi ${ }^{a}$
}

${ }^{a}$ Max Planck Institut für extraterrestrische Physik, Giessenbachstrasse, D-85748 Garching, Germany

${ }^{b}$ Universitäts-Sternwarte München, Scheinerstrasse 1, D-81679 München, Germany

${ }^{c}$ Technische Universität München, Fakultät für Physik, James-Franck-Strasse, 85748 Garching, Germany

${ }^{d}$ Excellence Cluster Universe, TUM, Boltzmannstrasse 2, 85748 Garching, Germany

${ }^{e}$ Max Planck Institut für Plasmaphysik, Boltzmannstrasse 2, 85748 Garching, Germany

${ }^{f}$ Dipartimento di Fisica e Astronomia, Universitá degli Studi di Firenze, Largo E. Fermi 2, 50125

Firenze, Italy

${ }^{g}$ Department of Astronomy, University of Washington, Box 351580, Seattle, WA 98195, USA

E-mail: xivryampe.mpg.de

\begin{abstract}
In this contribution we show that the host galaxies of NLS1s differ dramatically from those of Broad-Line Seyfert 1s (BLS1s). This leads us to propose that NLS1s represent a class of AGN in which the black hole growth is, and has always been, dominated by secular evolution. Our line of argument is as follows. Firstly, by looking at the properties of NLS1 hosts in the literature, we show that the evolution of NLS1s is currently mainly driven by secular processes, in contrast to BLS1s. Secondly, we study the bulges of NLS1 and BLS1 galaxies, and we find that statistically NLS1s have lower Sérsic indices $\left.\left(<n_{b}\right\rangle \sim 1.5\right)$ and less prominent bulges (bulge-to-total light ratio, $\langle B / T>\sim 0.2)$ than $\operatorname{BLS} 1 \mathrm{~s}\left(<n_{b}>\sim 2.5\right.$ and $\left.<B / T>\sim 0.4\right)$. This result indicates that NLS1 host bulges are pseudo-bulges and distinct from BLS1 bulges. The direct consequence of this result is that internal secular evolution must have dominated the past evolution of NLS1 hosts, possibly explaining their particular AGN properties.
\end{abstract}

Narrow-Line Seyfert 1 Galaxies and their place in the Universe - NLS1,

April 04-06, 2011

Milan Italy

\footnotetext{
* Speaker.

${ }^{\dagger}$ GOX acknowledges partial financial support from INAF.
} 


\section{Introduction}

While the main defining criteria of NLS1s with respect to BLS1s is the empirical threshold at $\operatorname{FWHM}\left(\mathrm{H}_{\beta}\right) \sim 2000 \mathrm{~km} \mathrm{~s}^{-1}$, the NLS1s have particular properties throughout the wavelength range. Trends and correlations have been identified using, first, small samples and, later, corroborated by larger surveys (e.g. [32, 33]). Many scenarios have been considered to explain these properties. The current, generally accepted, paradigm is that NLS1s have high accretion rates $\left(L / L_{\text {Edd }} \simeq 1\right.$, e.g. $[2,11]$ and reference therein) and low black hole masses (typically of order $10^{6} M_{\odot}$, e.g. $\left.[1,33,28]\right)$. While these scenarios can elucidate the nuclear properties of NLS1s, they hardly explain the origin of the fundamental differences between NLS1s and BLS1s and, in particular, that NLS1s appear to be more than just Seyfert 1s with narrow lines. A few key questions could be formulated as follows: which particular mechanisms would lead to the Eddington accretion rates commonly seen in NLS1s but observed less often in BLS1s? What causes the difference in black hole growth of NLS1s and BLS1s that results in low mass black holes in the former case? Could differing host galaxy evolution explain the differences between NLS1 and BLS1 galaxies?

In this contribution, rather than studying the active nuclei, we investigate the host galaxies of NLS1s and contrast their properties to those of BLS1s, pursuing the hypothesis that different host galaxy evolution could explain the differences between NLS1s and BLS1s. In particular, we explore the relative role of secular processes in the evolution of NLS1 and BLS1 galaxies. Reviewing the literature on the morphology and the star formation in NLS1 and BLS1 hosts, we emphasize, in section 2, the present-day differences in their respective host galaxies. We then explore, in section 3, the past evolution of NLS1 and BLS1 galaxies by studying their respective bulges. Indeed, this enables us to determine the main processes that have driven the evolution of NLS1 and BLS1 hosts, i.e. hierachical assembly or internal secular evolution. We therefore present here the results of a bulge-disk decomposition of samples of NLS1 and BLS1 galaxies, in particular their Sérsic indices and bulge-to-total light ratios. Using previously established criteria ([14, 7, 8]), we conclude that NLS1 hosts possess pseudo-bulges and, hence, have always been dominated by secular evolution. Finally, we shortly discuss in section 4 the implications of this result on our understanding of the NLS1 phenomenon.

\section{Present secular evolution}

The morphological differences between NLS1 and BLS1 galaxies can be summarized as follow: NLS1 host galaxies are likely to be strongly barred, much more than BLS1 ones, and their nuclear dust morphology is likely to be a grand-design spiral. Indeed, the results from [4, 25] both indicate that NLS1s are more likely to be strongly barred than BLS1s and that the fraction of barred Seyfert type 1 galaxies decreases with an increasing full width at half maximum of the broad component of the $\mathrm{H}_{\beta}$ emission line (from $\sim 65 \%$ to $\sim 25 \%$ between 500 and $\sim 2000 \mathrm{~km} \mathrm{~s}^{-1}$ ). In another study, [5] show that NLS1s usually have grand-design nuclear dust morphologies and are always associated with the presence of large-scale bars (see also [17]). But bars are also expected to drive gas inwards, e.g. [31]. Therefore, we also expect the presence of such asymmetries in the host galaxies to result in an enhanced star formation in the central kiloparsecs. 
In a recent paper and contribution to this conference, [30] specifically study the link between star formation in the central kiloparsecs versus the FWHM $\left(\mathrm{H}_{\beta}\right)$ in NLS1 and BLS1 host galaxies. After discussing carefully possible luminosity and distance effects, they conclude that NLS1s are associated with more intense star formation than BLS1s (with on average a star formation to AGN ratio $>2$ times larger in NLS1s).

Therefore, we can conclude that the current morphology and the central star formation of NLS1 host galaxies are distinguishable from other Seyfert galaxies. Indeed, in contrast to BLS1s, NLS1 galaxies are likely to be strongly barred and to show more intense central star formation. This is in line with the fact that bars are known to drive gas into the central kiloparsecs [29, 31], and that nuclear star formation is enhanced in barred galaxies, [13]. Hence, NLS1 galaxies show uninterrupted asymmetries able to drive the gas inwards from a few kiloparsecs to a few tens of parsecs. The particular strength of secular processes in NLS1s could therefore account for the high central star formation and presumably to the large Eddington rates observed in most of NLS1s.

\section{Past secular evolution}

One usually distinguishes two classes of bulges: pseudo- and classical bulges ${ }^{1}$. Pseudo-bulges are formed by internal secular processes such as bar instabilities, spiral structures, etc. (see [14], for a review), as opposed to galaxy mergers or external secular evolution (minor mergers, prolonged gas infall, etc.). As such, pseudo-bulges differ from classical bulges in that they were made slowly out of disk material while classical bulges are "merger-built" bulges. As pseudo-bulge retain memory of their disky origin, it is also possible to disentangle them from classical bulges.

Among the bulge properties, the Sérsic index of the bulge light profile is an established criterion to distinguish on average pseudo- from classical bulges (e.g., [14], [7]). Indeed, pseudo-bulges tend to have a lower Sérsic index similar to that of the outer disk and therefore would have a Sérsic index $n_{b} \sim 1-2$. Statistically, as shown by [7], pseudo-bulges have Sérsic indices of $n_{b}<2$ while classical bulges have $n_{b}>2$.

In addition to having low Sérsic index, pseudo-bulges are also found to be less prominent than classical bulges, in particular they tend to have a low bulge-to-total light ratio ([7, 8]).

Based on these criteria for the bulge classification of inactive galaxies, we perform a bulgedisk decomposition of a sample of NLS1s and BLS1s, and study the Sérsic index and the bulge prominence. We select NLS1 and BLS1 galaxies from an HST imaging survey of AGN, see [16]. Our final sample is composed of 9 genuine NLS1s and 18 BLS1s.

We compare in Fig. 1 the result obtained for our NLS1 and BLS1 samples with the distribution found by [7]. According to their mean Sérsic index, NLS1 host bulges $\left(<n_{b}>\sim 1.48\right)$ are pseudobulges $\left(<n_{b} \sim 1.69>\right.$ from [7]). BLS1 host bulges, on their side, are on average different from NLS1s but they also tend to have lower Sérsic indices $\left(<n_{b}>\sim 2.54\right)$ than classical bulges $(<$ $n_{b}>\sim 3.49$ from [7]). However, the mean bulge Sérsic index of inactive galaxies across the Hubble sequence have been found to be $\sim 2.5$, [15]. Therefore, the bulges of BLS1 hosts are likely to represent the overall bulge "population", while the bulges of NLS1 hosts are likely to be "pure" pseudo-bulges.

\footnotetext{
${ }^{1}$ Note however that bulges can have both a pseudo- and a classical component, see e.g. [24].
} 


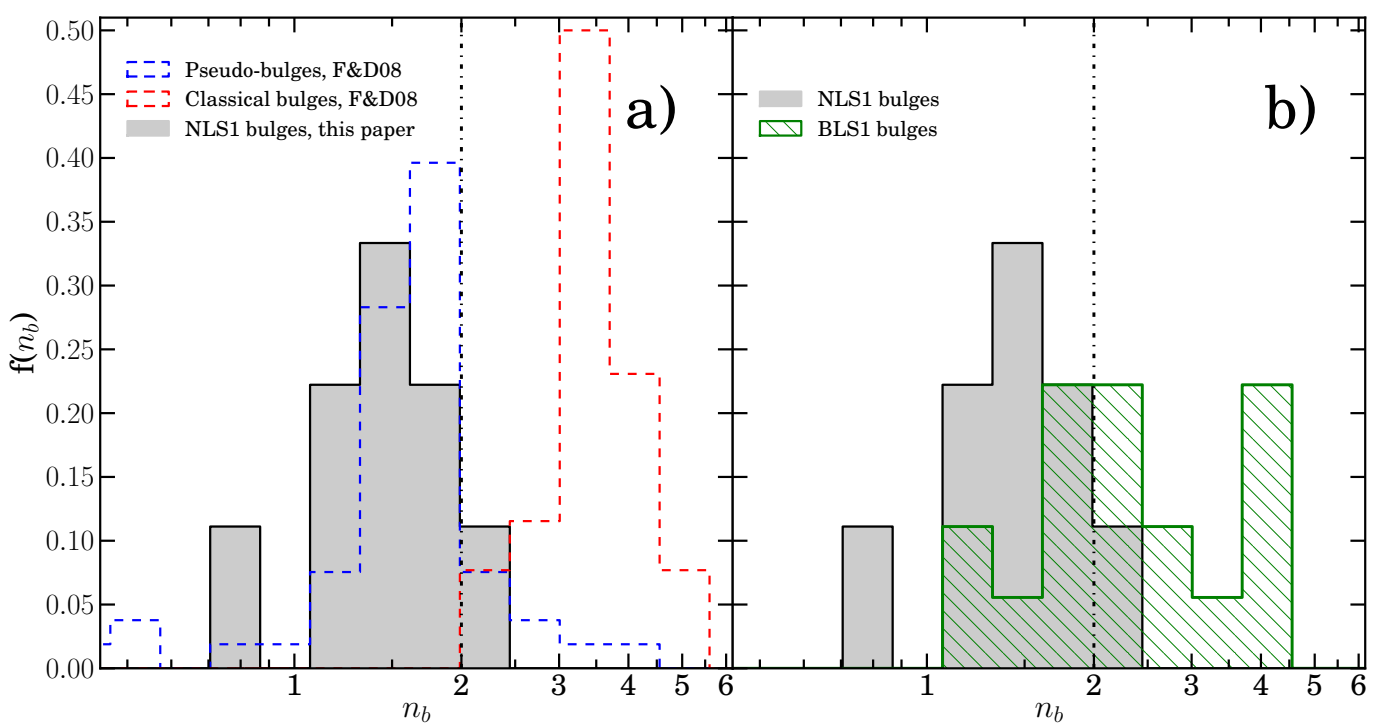

Figure 1: Histogram of bulge Sérsic indices, $n_{b}$. a) NLS1 host bulges (9 objects) from our sample compared to pseudo-bulges (53) and classical bulges (26, we do not include their sample of elliptical galaxies) from [7], F\&D08. b) NLS1 host bulges compared to BLS1s host bulges (18 objects). See also [26].

Building on our bulge-disk decomposition, we compute the bulge-to-total (B/T) light ratio using our fit parameters. We obtain respectively a median B/T of 0.39 for BLS1s and 0.17 for NLS1s. These median values and the distributions of B/T are consistent with NLS1s having pseudo-bulges and BLS1s having composite or classical bulges, see [7, 8]. Since the mean B/T ratio tends to decrease with the Hubble type, NLS1 galaxies tend to be of later type than BLS1 galaxies, typically Sbc versus Sa-Sab respectively, based on [18].

A few other studies support our conclusion about the bulges of NLS1s : [28, 10, 22]. When estimating the black hole masses of NLS1 galaxies, [28] performed a bulge-disk decomposition of several NLS1s and also find a low Sérsic index, $\left\langle n_{b}\right\rangle \sim 1.5$. In studying low black hole mass systems, [10] find that a large fraction of them have pseudo-bulges. And in fact, these objects are essentially NLS1s, [9]. Finally, in a recent paper, [22] also perform a bulge-disk decomposition of NLS1 and conclude that they have pseudo-bulges.

The consequence of NLS1 hosts having "pure" pseudo-bulges is that internal secular processes must have dominated the past evolution of NLS1 hosts. And therefore it is from this perspective that one should attempt to explain the particular AGN properties observed in NLS1 galaxies. 


\section{Conclusion and Implications}

From a review of the literature, we show that secular evolution in NLS1 galaxies is a powerful and on-going process on all scales, in contrast to BLS1 galaxies. To assess the role of secular processes in the past evolution of NLS1 galaxies, we examine their bulge properties by performing bulge-disk decompositions on NLS1 and BLS1 galaxies with archival HST images. The Sérsic index distribution and the prominence of the bulge that we found both indicate that, on average, NLS1 hosts have "pure" pseudo-bulges, in contrast to BLS1 galaxies. The consequence of this result is that internal secular processes must have dominated the past evolution of NLS1 hosts. We therefore conclude that NLS1s represent a class of AGN in which the black hole growth is, and has always been, dominated by secular evolution.

That NLS1s have been driven by such an evolutionary mode has several implications that can help our understanding of the NLS1 phenomenon.

One question concerning NLS1s that has received much attention is their location on the $\mathrm{M}_{\mathrm{BH}^{-}}$ $\sigma$ relation (e.g. $[19,12,20,21])$. Our results suggests that NLS1s lie in the same region as inactive galaxies with pseudo-bulges, that is scattered around and below the $\mathrm{M}_{\mathrm{BH}^{-}}-\sigma$. We should note, however, that this relation is not yet understood when secular processes drive the evolution of the bulge and the black hole. Therefore, while it is clear that the NLS1 black holes are still growing, we cannot predict where they will end up on the relation.

Finally, being driven by secular evolution, the prolonged disk accretion in NLS1s is likely to spin-up their central black hole. Indeed, by having a favoured direction related to the host structure, the angular momentum transferred to the black hole will eventually lead it to spin in the same direction and then spin up. But highly spinning BHs also lead to high radiative efficiency (because the last stable orbit is closer to the horizon of the BH). Therefore, secular evolution - as a plausible efficient way to spin up BHs - can explain the high Eddington ratio and the low black hole masses usually attributed to NLS1s. While there are a few high spin measurements of NLS1 BHs (see $[3,6,23,27]$ ), this picture is only suggestive and future $\mathrm{X}$-ray missions may be able to test this.

The content of this contribution and its implications are thoroughly presented in [26].

\section{References}

[1] Boller T., Brandt W. N., Fink H., Soft X-ray properties of narrow-line Seyfert 1 galaxies, A\&A, 305 (1996) 53

[2] Boroson T. A., Black Hole Mass and Eddington Ratio as Drivers for the Observable Properties of Radio-loud and Radio-quiet QSOs, ApJ, 565 (2002) 78.

[3] Brenneman L. W., A spectral survey of black hole spin in active galactic nuclei, $\mathrm{PhD}$ thesis, University of Maryland, College Park (2007).

[4] Crenshaw D. M., Kraemer S. B., Gabel J. R., The Host Galaxies of Narrow-Line Seyfert 1 Galaxies: Evidence for Bar-Driven Fueling, AJ, 126 (2003) 1690.

[5] Deo R. P., Crenshaw D. M., Kraemer S. B., The Host Galaxies of Narrow-Line Seyfert 1 Galaxies: Nuclear Dust Morphology and Starburst Rings, AJ, 132 (2006) 321. 
[6] Fabian A. C. et al., Broad line emission from iron $K$ - and L-shell transitions in the active galaxy 1H0707-495, Nature, 459 (2009) 540.

[7] Fisher D. B., Drory N., The Structure of Classical Bulges and Pseudobulges: the Link Between Pseudobulges and Sérsic Index, AJ, 136 (2008) 773.

[8] Gadotti D. A., Structural properties of pseudo-bulges, classical bulges and elliptical galaxies: a Sloan Digital Sky Survey perspective, MNRAS, 393 (2009) 1531.

[9] Greene J. E., \& Ho L. C., Active Galactic Nuclei with Candidate Intermediate-Mass Black Holes, ApJ, 610 (2004) 722 .

[10] Greene J. E., Ho L. C., \& Barth A. J., Black Holes in Pseudobulges and Spheroidals: A Change in the Black Hole-Bulge Scaling Relations at Low Mass, ApJ, 688 (2008) 159.

[11] Grupe D., A Complete Sample of Soft X-Ray-selected AGNs. II. Statistical Analysis, AJ, 127 (2004) 1799.

[12] Grupe, D., \& Mathur, S., $M_{B H^{-}} \sigma$ Relation for a Complete Sample of Soft X-Ray-selected Active Galactic Nuclei, ApJL, 606 (2004) L41.

[13] Ho L. C., Filippenko A. V., Sargent W. L. W., A Search for “Dwarf” Seyfert Nuclei. V. Demographics of Nuclear Activity in Nearby Galaxies, ApJ, 487 (1997) 591.

[14] Kormendy J., Kennicutt Jr. R. C., Secular Evolution and the Formation of Pseudobulges in Disk Galaxies, ARAA, 42 (2004) 603.

[15] Laurikainen E., Salo H., Buta R., \& Knapen J. H., Properties of bars and bulges in the Hubble sequence, MNRAS, 381 (2007) 401.

[16] Malkan M. A., Gorjian V., \& Tam R., A Hubble Space Telescope Imaging Survey of Nearby Active Galactic Nuclei, ApJS, 117 (1998) 25.

[17] Martini P., Regan M. W., Mulchaey J. S., Pogge R. W., Circumnuclear Dust in Nearby Active and Inactive Galaxies. II. Bars, Nuclear Spirals, and the Fueling of Active Galactic Nuclei, ApJ, 589 (2003) 774.

[18] Masters K. L., et al., Galaxy Zoo: dust in spiral galaxies, MNRAS, 404 (2010) 792.

[19] Mathur, S., Kuraszkiewicz, J., \& Czerny, B., Evolution of active galaxies: black-hole mass-bulge relations for narrow line objects, NewA, 6 (2001) 321.

[20] Mathur, S., \& Grupe, D., The Locus of Highly Accreting Active Galactic Nuclei on the $M_{B H}-\sigma$ Plane: Selections, Limitations, and Implications, ApJ, 633 (2005a) 688.

[21] Mathur, S., \& Grupe, D., Black hole growth by accretion, A\&A, 432 (2005b) 463.

[22] Mathur S., Fields D., Peterson B. M., \& Grupe D., Supermassive black holes, pseudobulges, and the narrow-line Seyfert 1 galaxies, ApJ, submitted [arXiv: 1102.0537 ].

[23] Miniutti G., Panessa F., de Rosa A., Fabian A. C., Malizia A., Molina M., Miller J. M., Vaughan S., An intermediate black hole spin in the NLS1 galaxy SWIFT J2127.4+5654: chaotic accretion or spin energy extraction?, MNRAS, 398 (2008) 255.

[24] Nowak N., Thomas J., Erwin P., Saglia R. P., Bender R., \& Davies R. I., Do black hole masses scale with classical bulge luminosities only? The case of the two composite pseudo-bulge galaxies NGC 3368 and NGC 3489, MNRAS, 403 (2010) 646. 
[25] Ohta K., Aoki K., Kawaguchi T., Kiuchi G., A Bar Fuels a Supermassive Black Hole?: Host Galaxies of Narrow-Line Seyfert 1 Galaxies, ApJS, 169 (2007) 1.

[26] Orban de Xivry G., Davies R., Schartmann M., Komossa S., Marconi A., Hicks E., Engel H., \& Tacconi L., The Role of Secular Evolution in the Black Hole Growth of Narrow-Line Seyfert 1 Galaxies, MNRAS, submitted [arXiv:1104.5023].

[27] Ponti G., et al., Relativistic disc reflection in the extreme NLS1 IRAS13224-3809, MNRAS, 406 (2010) 2591.

[28] Ryan C. J., De Robertis M. M., Virani S., Laor A., Dawson P. C., The Central Engines of Narrow-Line Seyfert 1 Galaxies, ApJ, 654 (2007) 799.

[29] Sakamoto K., Okumura S. K., Ishizuki S., Scoville N. Z., Bar-driven Transport of Molecular Gas to Galactic Centers and Its Consequences, ApJ, 525 (1999) 691.

[30] Sani E., Lutz D., Risaliti G., Netzer H., Gallo L. C., Trakhtenbrot B., Sturm E., Boller T., Enhanced star formation in narrow-line Seyfert 1 active galactic nuclei revealed by Spitzer, MNRAS, 403 (2010) 1246.

[31] Sheth K., Vogel S. N., Regan M. W., Thornley M. D., Teuben P. J., Secular Evolution via Bar-driven Gas Inflow: Results from BIMA SONG, ApJ, 632 (2005) 217.

[32] Williams R. J., Pogge R. W., Mathur S., Narrow-line Seyfert 1 Galaxies from the Sloan Digital Sky Survey Early Data Release, AJ, 124 (2002) 3042.

[33] Zhou H., Wang T., Yuan W., Lu H., Dong X., Wang J., Lu Y., A Comprehensive Study of 2000 Narrow Line Seyfert 1 Galaxies from the Sloan Digital Sky Survey. I. The Sample, ApJS, 166 (2006) 128. 